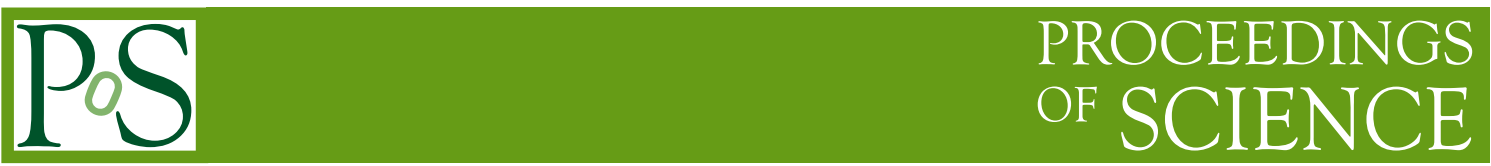

\title{
Minimum Bias Measurements at the LHC
}

\section{Deepak KAR ${ }^{* \dagger}$}

(University of Witwatersrand (SA))

E-mail: deepak.kar@cern.ch

\begin{abstract}
Inclusive charged particle measurements at hadron colliders probe the low-energy nonperturbative region of QCD. Pseudorapidity distributions of charged-particles produced in pp collisions at $13 \mathrm{TeV}$ have been measured by the CMS experiment. The ATLAS collaboration has measured the inclusive charged particle multiplicity and its dependence on transverse momentum and pseudorapidity in special data sets with low LHC beam current, recorded at a center-of-mass energy of $13 \mathrm{TeV}$. The measurements present the first detailed studies in inclusive phase spaces with a minimum transverse momentum of $100 \mathrm{MeV}$ and $500 \mathrm{MeV}$. The distribution of electromagnetic and hadronic energy in the very forward phase-space has been measured with the CASTOR calorimeters located at a pseudorapidity of -5.2 to -6.6 in the very forward region of CMS. The energy distributions are very powerful benchmarks to study the performance of MPI in hadronic interactions models at $13 \mathrm{TeV}$ collision energy. All measurements are compared with predictions of various Monte Carlo generator predictions and are found to provide strong constraints on these.
\end{abstract}

38th International Conference on High Energy Physics

3-10 August 2016

Chicago, USA

\footnotetext{
${ }^{*}$ Speaker.

${ }^{\dagger}$ On behalf of the ATLAS and CMS Collaborations
} 


\section{Introduction}

Several measurements using charged particle tracks and energy clusters have been performed by the ATLAS and CMS experiments $[1,2]$ at the Large hadron Collider (LHC) using the proton-proton $(p p)$ collisions data at a center-of-mass energy of $13 \mathrm{TeV}$ in so called minimum bias events. Minimum bias ${ }^{1}$ is a term used to describe the events selected with a minimally biased trigger, although the exact definition of the trigger is experiment dependent. They provide insight into the strong interaction in the low-energy, non-perturbative region of quantum chromodynamics (QCD). These interactions are modelled by Monte Carlo (MC) event generators with free parameters that can be tuned by such measurements. An accurate description of low-energy strong interaction processes is essential for simulating single pp interactions as well as the effects of multiple pp interactions (pile-up) at high instantaneous luminosity in hadron colliders. The MC models, with which the data is compared in this article, are briefly described in Tab. 1.

\begin{tabular}{ll}
\hline Generator (and tune) & Description \\
\hline Pythia8 [3] 4C [4] & MB+UE tune with CTEQ6L1 PDF [5] \\
Pythia8 Monash [6] & MB+UE tune with NNPDF2.3LO PDF [7] \\
Pythia8 CUETP8S1 [8] & CMS UE tune based on 4C \\
Pythia8 CUETP8M1 [8] & CMS UE tune based on Monash \\
Pythia8 A2 [9] & ATLAS MB/Central ET flow tune based on 4C \\
Herwig++ [10] UE-EE-5C [11] & UE tune with energy scaling using CTEQ6L1 PDF \\
\hline Epos LHC [12] & Based on Gribov's Pomeron exchange/collective flow \\
QGSJET-II [13] & approach [14], use LHC and fixed target experiment data \\
Sibyll [15] & to describe hadron and nuclear collisions \\
\hline
\end{tabular}

Table 1: Brief description of the MC generator models. The top part of the table depicts the parton shower models, while the bottom three models are all using the collective flow approach.

\section{Charged particle distributions}

Charged particle distributions at the beginning of LHC Run 1, at the center-of-mass energies of both $900 \mathrm{GeV}$ and $7 \mathrm{TeV}$ showed a significant discrepancy between data and predictions from then state-of-the art MC models [16]. Subsequently, the models were improved and new tunes were performed using the LHC data. So the big question before the start of the Run 2 was, will these improved models describe the data at a new, unprecedented collision energy.

In Fig. 1, the pseudorapidity distributions measured by CMS [17] and ATLAS [18, 19] are shown, with the transverse momenta, $p_{\mathrm{T}}$ threshold for charged particles increasing from zero in the leftmost plot, to $100 \mathrm{MeV}$ in the middle plot, and to $500 \mathrm{MeV}$ in the rightmost plot. Figure 2 shows the charged particle $p_{\mathrm{T}}$, multiplicity and event-by-event mean $p_{\mathrm{T}}$ against multiplicity distributions measured by ATLAS, with the top row showing distributions with $100 \mathrm{MeV} p_{\mathrm{T}}$ threshold, and bottom row showing distributions with $500 \mathrm{MeV} p_{\mathrm{T}}$ threshold. Overall Epos seems to predict the data the best, but most of the models show reasonable agreement. The ATLAS A2 tune does well for pseudorapidity distribution for the higher $p_{\mathrm{T}}$ threshold, but not for the lower one. For $p_{\mathrm{T}}$ and

\footnotetext{
${ }^{1}$ Minimum bias is often confused with underlying event (UE), which denotes the accompanying activity corresponding to an identified hard scatter, or with pile-up, which describes uncorrelated pp collisions in the same bunch-crossing.
} 
multiplicity distributions, except Monash, none of the models perform consistently well over the whole range. The mean $p_{\mathrm{T}}$ against multiplicity correlation depends on the colour reconnection, as seen by the total mismtach with QGSJET-II shape, which does not have any colour reconnection. Finally, in Fig. 3, the charged particle multiplicity measured by CMS and ATLAS as a function of center-of-mass energy is shown. About $20 \%$ increase in the multiplicity can be seen in going from 7 to $13 \mathrm{TeV}$. Most MC models are seen to get the energy extrapolation trend right.
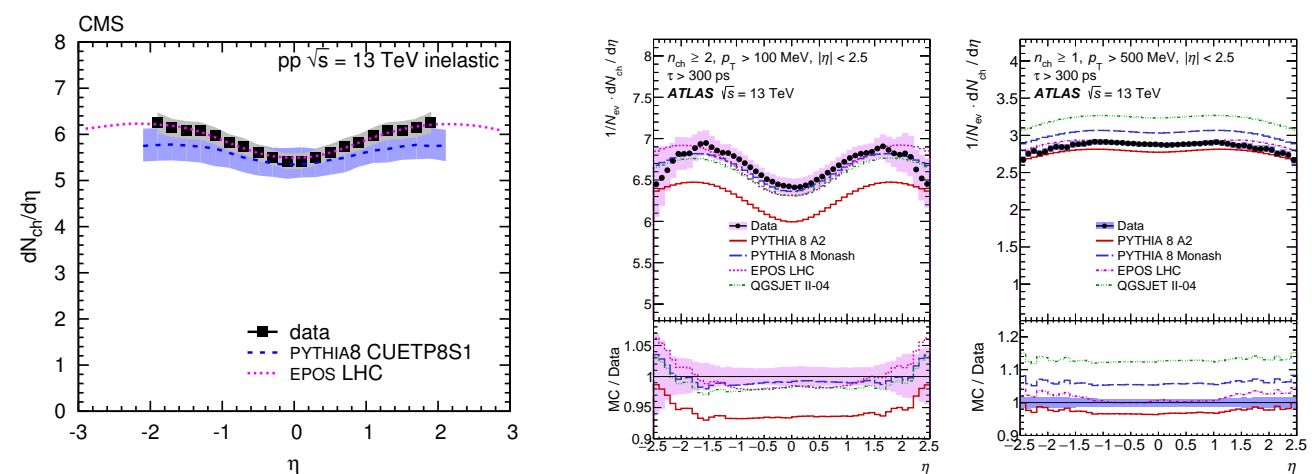

Figure 1: Charged particle pseudorapidity distributions measured by CMS (left) [17], and ATLAS with different $p_{\mathrm{T}}$ thresholds, increasing from middle [19] to right [18].
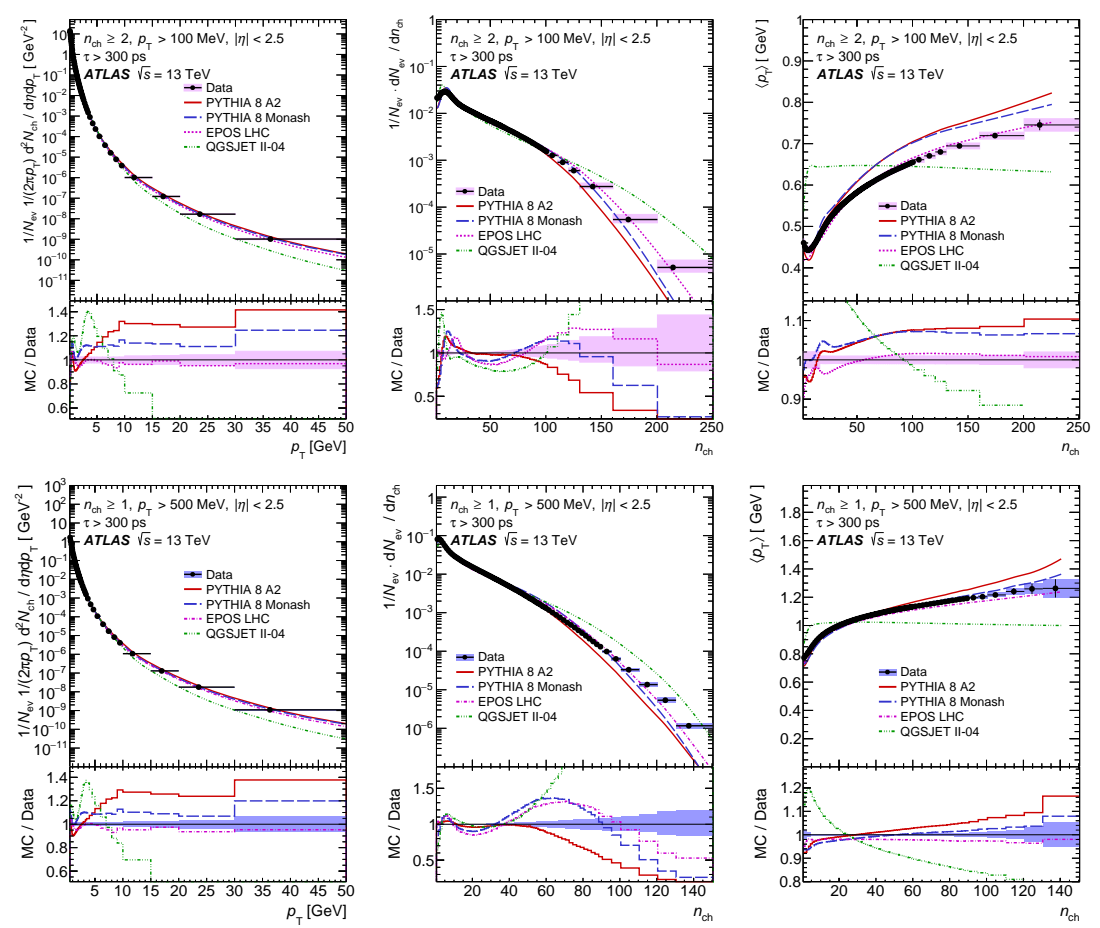

Figure 2: Charged particle distributions measured by ATLAS [18, 19] with $100 \mathrm{MeV}$ (top) and $500 \mathrm{MeV}$ (bottom) $p_{\mathrm{T}}$ thresholds. The left, middle and right columns respectively show transverse momentum, multiplicity and mean $p_{\mathrm{T}}$ against multiplicity distributions. 

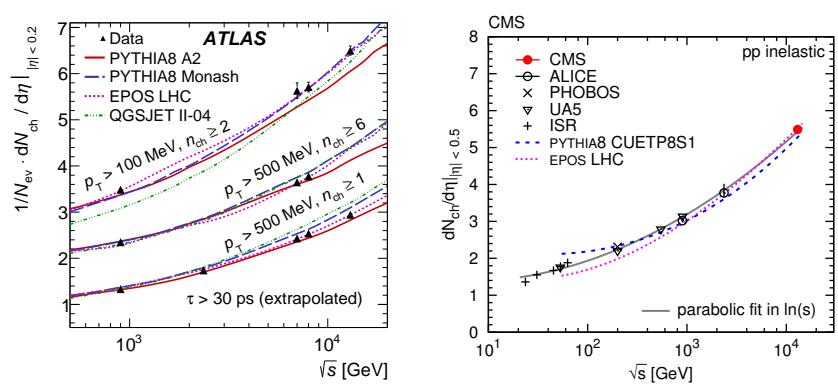

Figure 3: Dependence of charged particle multiplicity on center-of mass energy measured by ATLAS (left) [19], and CMS (right) [17].

\section{The A3 tune}

Although Pythia8 tunes are seen to model the charged particle distributions reasonably well, they were always seen to significantly overpredict the measured fiducial inelastic cross sections [20, 21]. All the previous tunes used the default Schuler and Sjöstrand (SS) [22] diffraction model. In the new ATLAS A3 tune the diffraction model was switched to Donnachie-Landshoff [23] model, and parameters controlling the multiple parton interaction (MPI) and colour reconnection were retuned [24]. This tune predicts an inelastic cross section much closer to the measured value (as seen in Tab. 2) and gives mostly similar level of agreement with minimum bias distributions.

\begin{tabular}{lrrr}
\hline & ATLAS data $(\mathrm{mb})$ & SS $(\mathrm{mb})$ & A3 $(\mathrm{mb})$ \\
\hline At $\sqrt{s}=13 \mathrm{TeV}$ & $68.1 \pm 1.4$ & 74.4 & 69.9 \\
At $\sqrt{s}=7 \mathrm{TeV}$ & $60.3 \pm 2.1$ & 66.1 & 62.3 \\
\hline
\end{tabular}

Table 2: Fiducial inelastic cross-section measured by ATLAS [20, 21] compared with A3 and Schuler and Sjöstrand (SS) model predictions. The SS model is used in both A2 and Monash tunes.

\section{Forward energy flow}

The measurements using charged and neutral calorimeter energy clusters offer complementary information to only charged particle distributions as described earlier. The CMS collaboration measured the energy flow in the pseudorapidity range $3.15<|\eta|<6.6$, and compared the data to different generator predictions [25], as shown in the left and the middle plots of Fig. 4. Models in general perform worse in the more forward region, but a large spread in predictions can be seen. Same distributions measured in non-single diffractive events are described better by models, indicating that the modelling of diffraction is still not very accurate. In the right plot of Fig. 4, the transverse energy flow as a function of shifted pseudorapidity is shown. This observable is sensitive to longitudinal scaling behaviour, whereas the data is seen to be consistent across a wide range of collision energies, and becomes independent of collision energy at the beam fragmentation region.

Figure 5 and Fig. 6 show the event-by-event energy deposition in the very forward region [26], $-5.2<\eta<-6.6$, measured by CMS, using the CASTOR detector. The results are compared to different MC models, and significant differences can be seen. Generally models do worse at the diffraction-dominated soft part of the distributions in Fig. 5. In Fig. 6 the energy deposit in 
electromagnetic and hadronic calorimeters are looked at separately. Models overall perform better for the electromagnetic case, which is more sensitive to MPI. Cosmic shower models do somewhat better for the hadronic component, but none predicts the entire shape well.
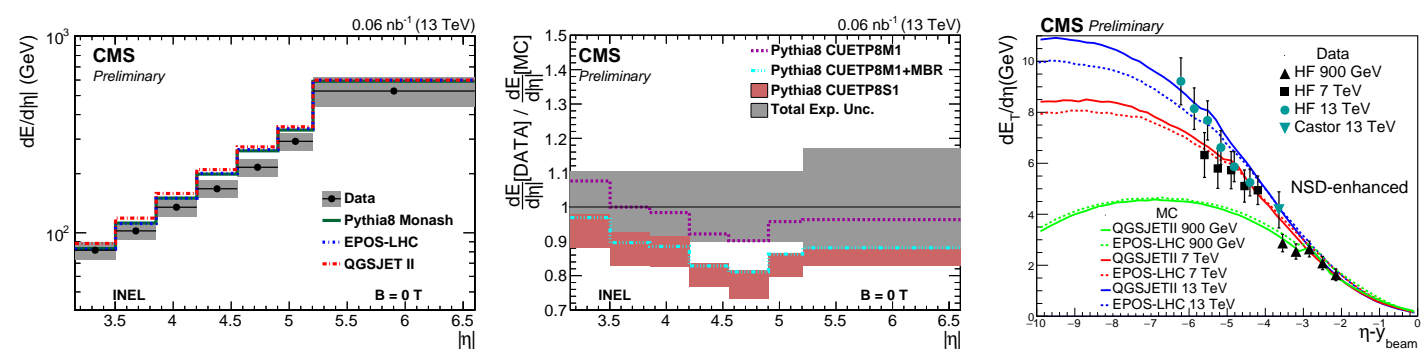

Figure 4: Forward energy flow distributions measured by CMS [25], and compared to collective flow based models (left), parton shower models (middle), and plotted as a function of shifted pseudorapidity (right).
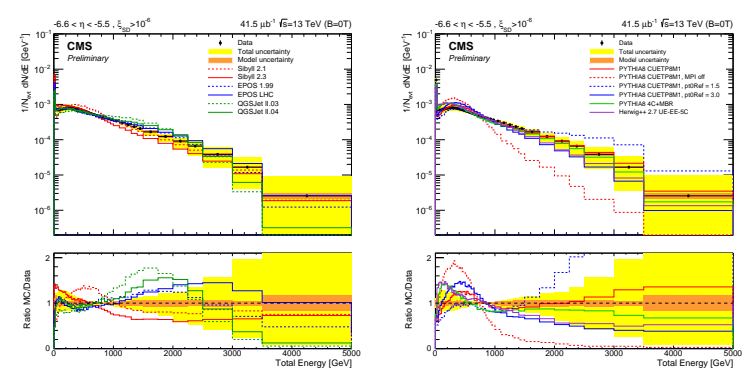

Figure 5: Very forward energy flow distributions measured by CMS [26], and compared to collective flow based models (left), and parton shower models (right).
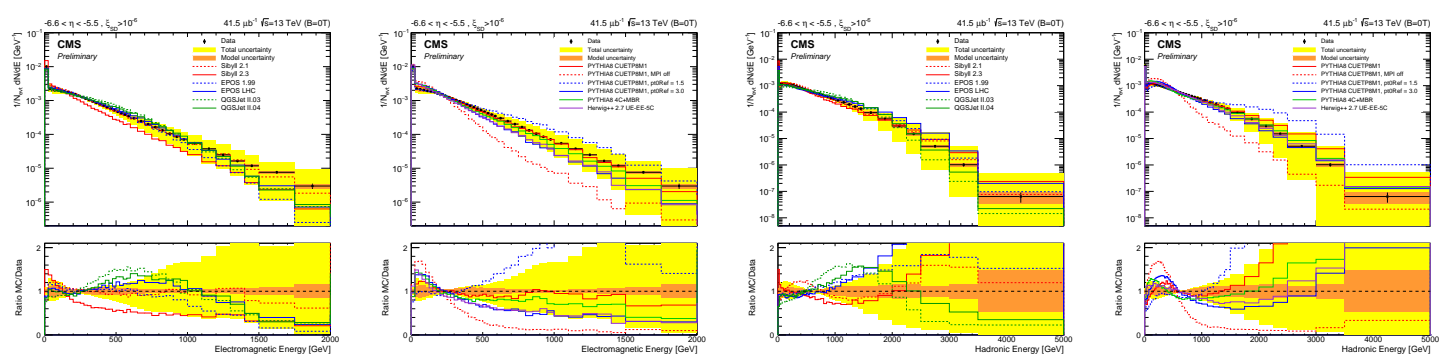

Figure 6: Very forward energy flow distributions measured by CMS [26], separated into electromagnetic (left two), and hadronic components (right two).

\section{Summary}

A wide range of minimum bias measurements, both with charged and neutral particles have been performed with LHC Run 2 data. While none of the models considered is perfect for all observables and full ranges, some do a reasonable job. That indicates the phenomenologically modelled center-of-mass energy dependence of MPI is not totally off as was the case in Run 1, where the extrapolation factor was larger. These results are important for pile-up modelling, and constraining the Monte Carlo event generators. 


\section{References}

[1] ATLAS Collaboration, The ATLAS Experiment at the CERN LHC, JINST 3, S08003 (2008).

[2] CMS Collaboration, The CMS experiment at the CERN LHC, JINST 3, S08004 (2008).

[3] T. Sjöstrand et al., An Introduction to PYTHIA 8.2, Comput. Phys. Commun. 191, 159 (2015).

[4] R. Corke et al., Interleaved Parton Showers and Tuning Prospects, JHEP 1103, 032 (2011).

[5] H. L. Lai et al., Parton Distributions for Event Generators, JHEP 1004, 035 (2010).

[6] P. Skands et al., Tuning PYTHIA 8.1: the Monash 2013 Tune, EPJC 74, no. 8, 3024 (2014).

[7] R. D. Ball et al., Parton distributions with LHC data, Nucl. Phys. B 867, 244 (2013).

[8] CMS Collaboration, Event generator tunes obtained from underlying event and multiparton scattering measurements, EPJC 76, no. 3, 155 (2016).

[9] ATLAS Collaboration, Further ATLAS tunes of PYTHIA6 and Pythia 8, ATL-PHYS-PUB-2011-014.

[10] M. Bahr et al., Herwig++ Physics and Manual, EPJC 58, 639 (2008).

[11] S. Gieseke et al., Colour reconnections in Herwig ++, EPJC 72, 2225 (2012).

[12] T. Pierog et al., EPOS LHC: Test of collective hadronization with data measured at the CERN LHC, PRC 92, no. 3, 034906 (2015).

[13] S. Ostapchenko, Monte Carlo treatment of hadronic interactions in enhanced Pomeron scheme: I. QGSJET-II model, PRD 83, 014018 (2011).

[14] H. J. Drescher et al., Parton based Gribov-Regge theory, Phys. Rept. 350, 93 (2001).

[15] E. J. Ahn et al., Cosmic ray interaction event generator SIBYLL 2.1, PRD 80, 094003 (2009).

[16] ATLAS Collaboration, Charged-particle multiplicities in pp interactions measured with the ATLAS detector at the LHC, New J. Phys. 13, 053033 (2011).

[17] CMS Collaboration, Pseudorapidity distribution of charged hadrons in proton-proton collisions at $\sqrt{s}$ = $13 \mathrm{TeV}$, PLB 751, 143 (2015).

[18] ATLAS Collaboration, Charged-particle distributions in $\sqrt{s}=13 \mathrm{TeV}$ pp interactions measured with the ATLAS detector at the LHC, PLB 758, 67 (2016).

[19] ATLAS Collaboration, Charged-particle distributions at low transverse momentum in $\sqrt{s}=13 \mathrm{TeV}$ pp interactions measured with the ATLAS detector at the LHC, EPJC 76, no. 9, 502 (2016).

[20] ATLAS Collaboration, Measurement of the Inelastic Proton-Proton Cross Section at $\sqrt{s}=13 \mathrm{TeV}$ with the ATLAS Detector at the LHC, arXiv:1606.02625 [hep-ex].

[21] ATLAS Collaboration, Measurement of the Inelastic Proton-Proton Cross-Section at $\sqrt{s}=7$ TeV with the ATLAS Detector, Nature Commun. 2, 463 (2011).

[22] G.A Schuler et al., Hadronic diffractive cross sections and the rise of the total cross section, PRD 49, 2257 (1994).

[23] A. Donnachie et al., Elastic Scattering and Diffraction Dissociation, Nucl. Phys. B 244, 322 (1984).

[24] ATLAS Collaboration, A study of the Pythia 8 description of ATLAS minimum bias measurements with the Donnachie-Landshoff diffractive model, ATL-PHYS-PUB-2016-017.

[25] CMS Collaboration, Measurement of the pseudorapidity dependence of the energy and transverse energy density in pp collisions at $\sqrt{s}=13 \mathrm{TeV}$ with CMS, CMS-PAS-FSQ-15-006.

[26] CMS Collaboration, Measurement of the energy distribution in the very forward direction at $13 \mathrm{TeV}$ with CMS, CMS-PAS-FSQ-16-002. 